\title{
INFLUÊNCIA DO GRAU DE HIDROFOBICIDADE DE SUPORTES POLIMÉRICOS COM MORFOLOGIA CASCA-NÚCLEO NA IMOBILIZAÇÃO DA LIPASE B DE Candida antarctica(CAL-B)
}

\author{
L. C. M.CIRILO ${ }^{1}$, M. C. C. PINTO ${ }^{2}$, D. M. G. FREIRE ${ }^{1}$, J. C. PINTO ${ }^{2}$ \\ ${ }^{1}$ Universidade Federal do Rio de Janeiro, Instituto de Química \\ ${ }^{2}$ Universidade Federal do Rio de Janeiro, Programa de Engenharia Química/COPPE \\ E-mail para contato: pinto@peq.coppe.ufrj.br
}

\begin{abstract}
RESUMO - O emprego de enzimas na forma imobilizada tem crescido nos últimos anos, em função, principalmente, da possibilidade de reutilização de tais enzimas. Dessa forma, objetivando desenvolver biocatalisadores mais eficientes, partículas poliméricas com morfologia casca-núcleo, apresentando diferentes graus de hidrofobicidade na casca, foram sintetizadas. Em uma etapa seguinte,tais partículas foram empregadas como suporte na imobilização da lipase B de Candida antarctica(CAL-B). Observou-se, então, que ao alterar a composição da casca durante a polimerização eram obtidas partículas com diferentes polaridades. Notou-se também que os biocatalisadores apresentaram maiores atividades de esterificação, quando suportes com maior caráter hidrofóbico foram empregados no processo de imobilização; possivelmente, em decorrência da maior exposição do sítio ativo das enzimas. No entanto, ao analisar as atividades de hidrólise dos biocatalisadores, percebeu-se também um aumento de atividade enzimática quando suportes mais hidrofílicos foram empregados, indicando a presença de limitações difusionais neste tipo de reação. Percebeu-se, assim, que o desempenho dos biocatalisadoresproduzidos dependenão somente das características dos suportes empregados no processo de imobilização, mas também do meio reacional onde o biocatalisador é empregado.
\end{abstract}

\section{INTRODUÇÃO}

A utilização de enzimas em processos industriais tem despertado grande interesse comercial, uma vez que as enzimas são catalisadores naturais e, muitas vezes, apresentam alta especificidade, seletividade e eficiência em condições reacionaisbrandas. Assim, as enzimas vêm sendo amplamente empregadas em diversos setores, comona áreaalimentícia, farmacêutica, química, têxtil, de biodiesel, dentre outras (Castroet al., 2008; Cunhaet al., 2014).

No entanto, há algumas desvantagens relacionadas ao emprego de tais enzimas na forma solúvel, principalmente a dificuldade de recuperá-las ao final do processo. Como as enzimas são catalisadores, em geral, caros, e de difícil produção em estado puro, torna-se economicamente conveniente reutilizá-las. Dessa forma, diferentes técnicas de imobilização enzimática vêm sendo 


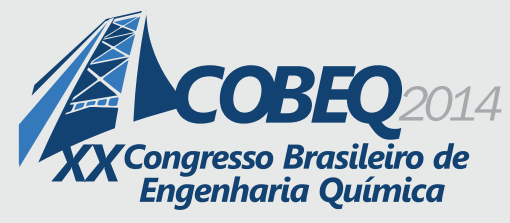

19 a 22 de outubro de 2014

Florianópolis/SC

desenvolvidas, objetivando garantir a recuperação das enzimas ao final de cada processo e também aumentar a estabilidade enzimática (Villeneuveet al., 2000). No entanto, vale destacar que, para empregar um determinado material como suporte para imobilização enzimática, este deverá apresentar algumas características básicas, como ser insolúvel em meio aquoso;ser estável mecanicamente; apresentar porosidade adequada; interagir com a enzima de forma a evitar sua dessorção, mas sem inativá-la; apresentarbaixo custo de produção (Freire, 1996).

A técnica de polimerização em suspensão e emulsão simultâneas tem recebido, então, especial destaque. A partir deste processo de polimerização é possível sintetizar partículas com morfologia casca-núcleoporosas, com área específica expressiva, apresentando grupamentos funcionais na casca sem haver a necessidade de etapas adicionais de funcionalização. Essa técnica engloba uma etapa inicial (a polimerização em suspensão), na qual ocorre a formação do núcleo polimérico. No momento em quereação atinge uma determinada conversão, novos constituintes são alimentados ao reator, iniciando-se a etapa de polimerização em emulsão. Os processos em emulsão e suspensão são, então, conduzidos simultaneamente. Por apresentar um caráter pegajoso, o núcleo captura as partículas geradas na polimerização em emulsão, havendo a formação da casca polimérica (Lenzi et al., 2003).

Nesse contexto, o presente trabalho visou à produção de diferentes biocatalisadores com potencial aplicação na síntese de biodiesel e na resolução de misturas racêmicas. Para isso, inicialmente, diferentes reações de polimerização em suspensão e emulsão simultâneas foram realizadas. Os núcleos foram produzidos a partir da polimerização em suspensão do estireno. Em seguida, os componentes da emulsão foram alimentados, havendo a formação da casca polimérica. Nesta etapa da reação, diferentes comonômeros foram copolimerizados com estireno, gerando diferentes partículas poliméricas com morfologia casca-núcleo, apresentando diferentes graus de hidrofobicidade na casca. Em uma etapa subsequente, tais partículas foram empregadas como suporte na imobilização da lipase B de Candida antarctica (CAL-B). O comportamento cinético de cada uma das imobilizações realizadas e as atividades de hidrólise e de esterificação de cada um dos biocatalisadores produzidos (enzima já imobilizada no suporte) foram avaliados.

\section{MATERIAIS E MÉTODOS}

\subsection{Materiais}

Todos os reagentes foram empregados sem nenhumaetapa de purificação. A Lipozyme ® CALB L, lipase B de Candida antarctica (CAL-B), na forma solúvel, foi fornecida pela empresa Novozymes. O substrato utilizado para a determinação das atividades de hidrólise foi o $p$-nitrofenil laurato ( $p$-NPL), fornecido pela Fluka, com pureza mínima de $98 \%$ p/p. O etanol PA, fornecido pela Vetec Química Fina, com uma pureza mínima de 99,8\% p/p, foi utilizado para a lavagem dos suportes poliméricos e também como substrato nas reações de esterificação. $\mathrm{O}$ ácido oleico foi fornecido pela Vetec Química Fina (com pureza mínima de $98 \%$ p/p) e foi usado como substrato nas reações de esterificação. Os monômeros empregados nas reações de polimerização foram: estireno, fornecido pela Sigma Aldrich, com pureza mínima de 99,5\% p/p; divinilbenzeno (DVB), fornecido pela Merck com pureza mínima de $99 \%$ p/p; 1-octeno,fornecido pela Merck, com pureza mínima de 97\% p/p; 


\section{9 a 22 de outubro de 2014 \\ Florianópolis/SC}

benzoato de vinila, fornecido pela Sigma Aldrich, com pureza mínima de $99 \%$ p/p; cardanol, fornecido pela Resibrás, com pureza mínima de $95,4 \%$ p/p. Outros reagentes e solventes utilizados na purificação e caracterização das partículas poliméricas e dos biocatalisadores eram de grau analítico.

\subsection{Preparação dos suportes}

Os suportes poliméricos com morfologia casca-núcleo foram sintetizados apartir da técnica de polimerização em suspensão e emulsão simultâneas, como descrito em alguns trabalhos da literatura (Lenzi et al., 2003; Besteti, 2009; Pinto, 2013; Cunha et al., 2014). As reações foram realizadas em reator de vidro de $1 \mathrm{~L}$ encamisado, acoplado a um banho termostático, usado para manter a temperatura da camisa a $85^{\circ} \mathrm{C}$. Para a síntese do núcleo, na etapa de suspensão, foi empregado apenas o estireno como monômero. No entanto, visando à alteração do grau de hidrofobicidade da casca, diferentes monômeros foram empregados na etapa de polimerização em suspensão e emulsão simultâneas(estireno, divinilbenzeno, cardanol, benzoato de vinila e 1-octeno). A partir destas reações, foram obtidos suportes com morfologia (casca/núcleo) com núcleo de poliestireno (PS) e diferentes composições na casca: poliestireno (PS), poli(estireno-co-benzoato de vinila) (PS-co-PBV), poli(estireno-co-octeno) (PS-co-PO), poli(estireno-co-cardanol) (PS-co-PC) e poli(estireno-codivinilbenzeno) (PS-co-PDVB). A composição dos comonômeros adicionados à casca foi de $5 \% \mathrm{p} / \mathrm{p}$ em relação à carga monomérica total da casca.Após o término das reações, as partículas foram filtradas, lavadas e secas.

\subsection{Caracterização dos suportes}

As partículas poliméricas casca-núcleo foram caracterizadas quanto a sua morfologia, por meio de estudos de microscopia eletrônica por varredura (MEV), usando ummicroscópio Fei Company, modelo Quanta 2000. As partículaspassaram por um processo prévio de metalização. Para caracterizar a área específica, o diâmetro médio de poros e o volume específico de poros dos suportesproduzidos, foi utilizado o equipamento ASAP 2020, fornecido pela Micrometics, usando-se nitrogênio como gás adsorvente (fisissorção) e empregando-se o modelo BET e o método BJH. A determinaçãodo ângulo de contato, importante para diferenciar o grau de polaridade das partículasproduzidas, foi realizada em um tensiômetro Kruss Process, modelo K100, utilizando a água como meio de referência.

\subsection{Imobilização enzimática}

Os procedimentos de imobilização enzimática e de dosagem das atividades de hidrólise e de esterificação dos biocatalisadores (enzimas já imobilizadas) são descritos em diferentes estudos da literatura (Cunha, 2011; Pinto, 2013; Cunha et al., 2014). Conforme os estudos apresentados, aprimeira etapa do processo de imobilização consistiu na lavagem dos suportes,buscando-se diminuir o grau de hidrofobicidade dos mesmos e facilitar a penetração da solução enzimática para seu interior. Dessa forma, $1 \mathrm{~g}$ de cada suporte foi colocado em contato com $10 \mathrm{ml}$ de etanol por um período de 30min.Em seguida, os suportes foram filtradose lavados com água destilada até completa remoção de etanol. Por fim, os suportes foram lavados com uma solução de fosfato de sódio (5mM, pH 7), filtrados e armazenadosna geladeira. 


\section{9 a 22 de outubro de 2014 \\ Florianópolis/SC}

Após a lavagem, a1g de cada suporte foram adicionados $10 \mathrm{ml}$ da solução enzimática diluída, com atividade inicial de $100 \mathrm{U} / \mathrm{g}_{\text {suporte }}$ A imobilização enzimática ocorreu por um período de $25 \mathrm{~h}$, com uma velocidade de agitação de $250 \mathrm{rpm}$ a $4{ }^{\circ} \mathrm{C}$. A cinética de imobilização foi acompanhada por meio da dosagem da atividade hidrolítica da enzima ainda presente na fase solúvel ao longo da imobilização, empregando-se o p-nitrofenil laurato, como substrato (Cunha, 2011; Pinto, 2013; Cunha et al., 2014).

\subsection{Atividade hidrolítica}

Após a imobilização, os biocatalisadores (enzima já imobilizada) foram avaliados quanto à capacidade de catalisar reações de hidrólise. A atividade de hidrólise da enzima imobilizada foi determinada por método espectrofotométrico a $30{ }^{\circ} \mathrm{C}$ (comprimento de onda de $412 \mathrm{~nm}$ ), usando $p$ nitrofenil laurato como substrato. A reação era iniciada pela adição do biocatalisador a um meio contendo a solução de substrato e tampão fosfato de sódio $(25 \mathrm{mM}, \mathrm{pH} 7)$. Uma unidade internacional (UI) foi definida como a quantidade de enzima necessária para formar $1 \mu$ mol de p-nitrofenol por minuto nas condições de ensaio (Cunha, 2011; Pinto, 2013; Cunha et al., 2014). As reações de hidrólise foram realizadas em triplicata e o erro experimental foi calculado com grau de confiança de $95 \%$.

\subsection{Atividade de esterificação}

Os biocatalisadores também foram avaliados quanto à capacidade de catalisar reações de esterificação. Para tal, 0,2 $\mathrm{g}$ de biocatalisador foram adicionados a um reator encamisado contendo uma mistura de 10,1 ml de ácido oleico e 1,9 ml de etanol (substratos das reações de esterificação). As reaçõesforam realizadas a $40{ }^{\circ} \mathrm{C}$ e a $250 \mathrm{rpm}$. Ao longo da reação, alíquotas de $100 \mu \mathrm{l}$ do meio reacional foram recolhidas e tituladas comuma solução de $\mathrm{NaOH} 0,02 \mathrm{M}$, a fim de determinar a concentração de ácido oleico ainda presente no meio reacional. Uma unidade de atividade da lipase (UI) foi definida como a quantidade de enzima necessária para formar $1 \mu \mathrm{mol}$ de oleato de etila por minuto nas condições reacionais utilizadas (Cunha, 2011; Pinto, 2013; Cunha et al., 2014). Algumas reações foram realizadas em triplicata eo erro experimental foi calculado com grau de confiança de $95 \%$.

\section{RESULTADOS E DISCUSSÃO}

Como mencionado anteriormente, para avaliar a influência da composição da casca polimérica dos suportes casca-núcleo na imobilizaçãoCAL-B, foram produzidas partículas com diferentes composições epolaridades na casca.A partir das análises das micrografias, apresentadas na Figura 1, é possível observar que os suportespossuem estrutura casca-núcleo e um aspecto floculento, resultado da deposição das partículas geradas na etapa da polimerização em emulsão sobre os núcleos de PS. Além disso, é possível perceber que em algumas partículas o recobrimento do núcleo foi menos uniforme, como é o caso das partículas com casca de PS-co-PC.

$\mathrm{Na}$ Tabela 1 são apresentados os resultados de diâmetro médio de poro e área específica das partículas produzidas. Nota-se que os valores de diâmetro médio de poro dos suportes sintetizados 
estão na faixa de 217,8 a 378,0 Å. Ou seja, não há nenhum impedimento espacial para o acesso da CAL-B ao interior dos poros dos suportes, uma vez que esta possui dimensões de $30 \AA$ x $40 \AA$ x $50 \AA$ (Uppenberg et al., 1994). Observa-se também a existência de uma relação inversa entre área específica e diâmetro médio de poro, indicando que ocorre a aglomeração das partículas geradas na fase da emulsão sobre os núcleos de PS (gerados na fase da polimerização em suspensão). Percebe-se também que, apesar da pequena alteração na composição da casca, partículas com diferentes graus de hidrofobicidade foram produzidas, uma vez que os ângulos de contato obtidos variaram na faixa de 71,3 a $91,8^{\circ}$.
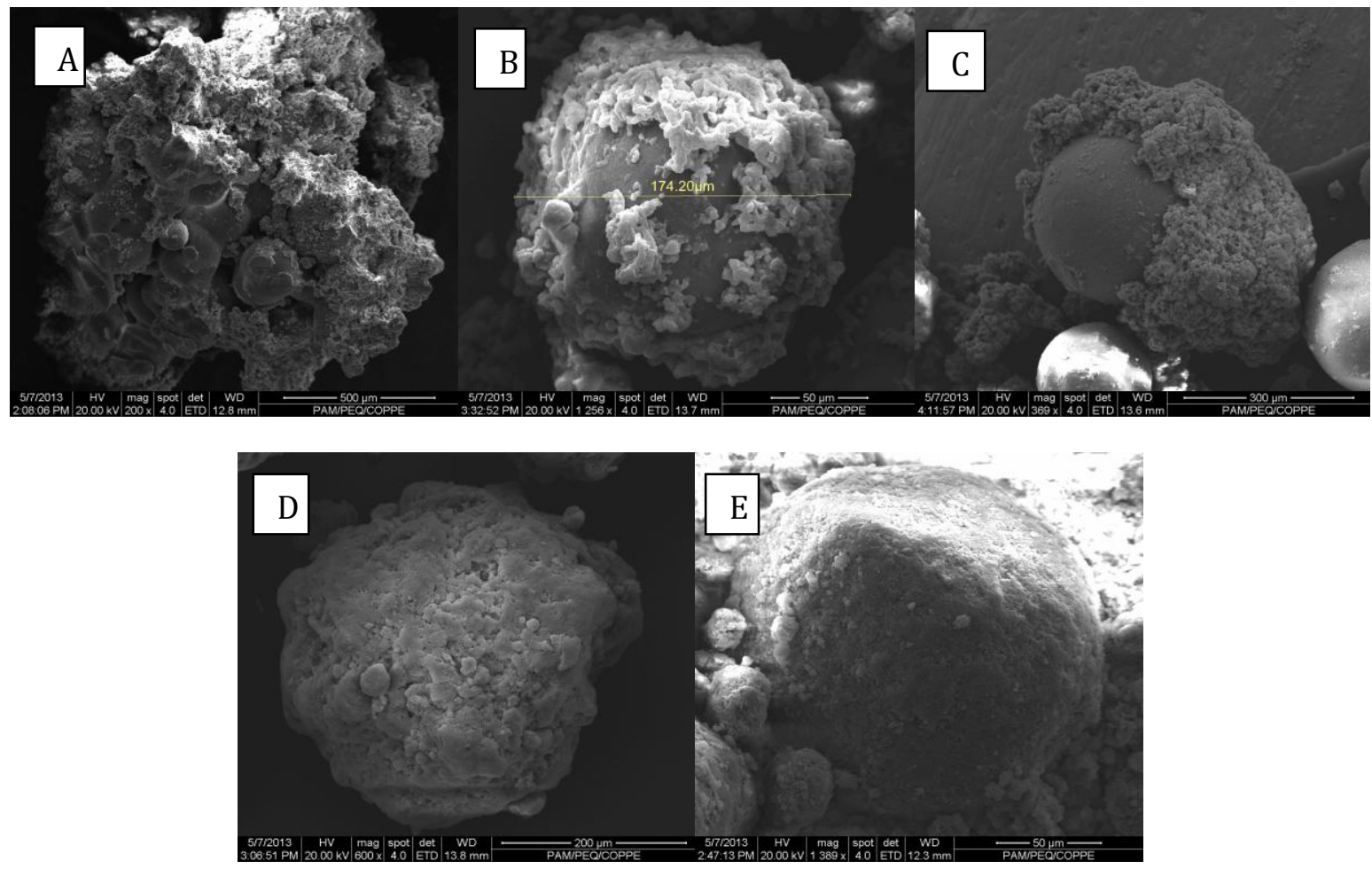

Figura 1 - A) PS/PS; B) PS-co-PBV/PS; C) PS-co-PC/PS; D) PS-co-PO/PS; E) PS-co$\mathrm{PDVB} / \mathrm{PS}$.

Na Figura 2 são mostrados os perfis cinéticos das imobilizações enzimáticas nos diferentes suportes produzidos. É possível observar que o suporte com casca de PS-co-PO apresentou maior velocidade de adsorção da lipase nos instantes iniciais do processo de imobilização. Nota-se também que quando o suporte de PS-co-PBV/PS foi empregado, uma maior quantidade de enzima foi adsorvida (menor quantidade de enzima presente no sobrenadante), seguido pelos suportes de PS-coPC/PS e PS-co-PO/PS. Esses resultados mostram que, dentre outros fatores, a existência de grupamentos polares na casca dos suportes facilita a imobilização da CAL-B, uma vez que a imobilização é realizada em meio polar, havendo maior facilidade do meio enzimático difundir para o interior dos poros. 
Tabela 1 - Características superficiais dos suportes produzidos.

\begin{tabular}{|c|c|c|c|}
\hline Suporte casca-núcleo & $\begin{array}{c}\text { Área específica } \\
\left(\mathrm{m}^{2} / \mathrm{g}\right)\end{array}$ & $\begin{array}{c}\text { Diâmetro médio de poro } \\
(\AA)\end{array}$ & $\begin{array}{c}\AA \\
\text { Ângulo de contato } \\
\left({ }^{\circ}\right)\end{array}$ \\
\hline PS-co-PDVB/PS & 18,3 & 217,8 & $84,0 \pm 1,7$ \\
\hline PS-co-PO/PS & 5,6 & 292,6 & $71,9 \pm 0,9$ \\
\hline PS-co-PC/PS & 3,2 & 264,8 & $78,4 \pm 1,7$ \\
\hline PS/PS & 1,6 & 357,5 & $91,8 \pm 0,7$ \\
\hline PS-co-PBV/PS & 1,2 & 378,0 & $71,3 \pm 2,3$ \\
\hline
\end{tabular}

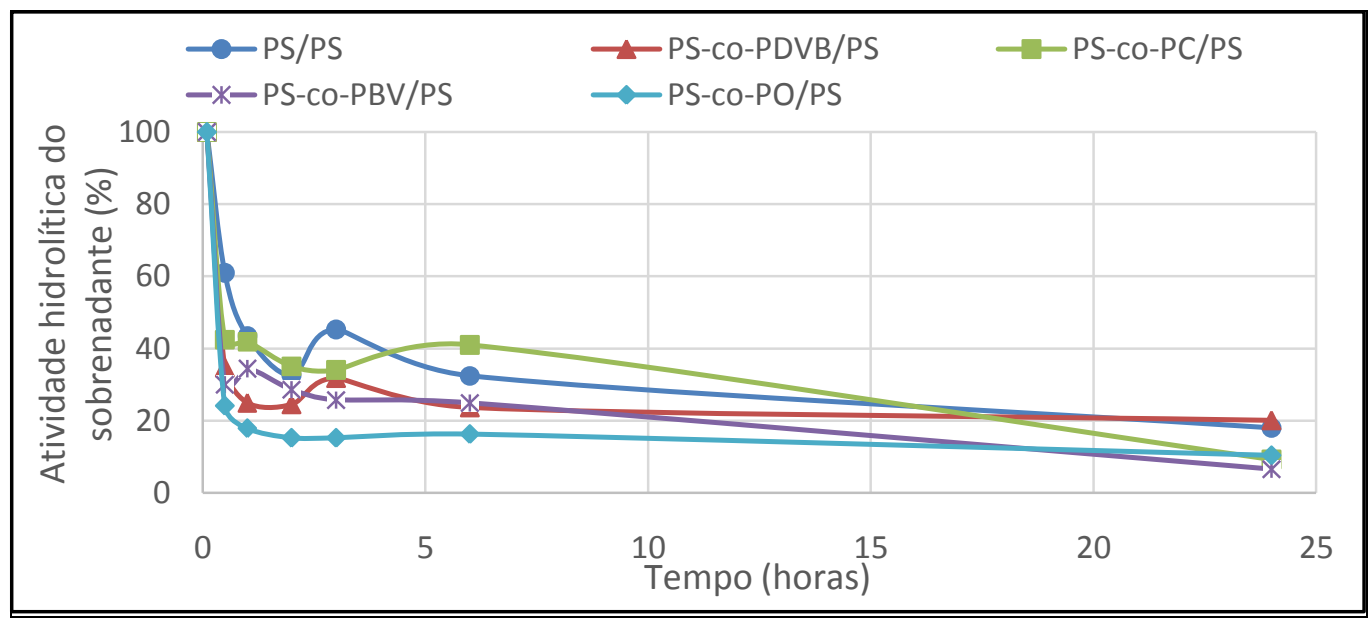

Figura 2 - Perfil cinético da imobilização da CAL-Bnos diferentes suportes produzidos.

A partir dos resultados apresentados na Tabela 2, nota-se que os biocatalisadores apresentam uma baixa atividade hidrolítica, quando comparadas às atividades de esterificação, confirmando resultados obtidos em estudos anteriores para suportes de PS/PS (Pinto, 2013). A baixa atividade hidrolítica é resultado das características da própria CAL-B, que é comumente empregada para catalisar reações de esterificação. Na Figura 3 é apresentada a influência do grau de hidrofobicidade dos suportes no desempenho dos biocatalisadores.

Ao observar a Figura 3-A), nota-se que quando suportes com maior carácter hidrofóbico são empregados, há uma tendência de aumento da atividade de hidrólise do biocatalisador; muito provavelmente, em função da maior exposição do centro ativo da CAL-B (Fernandez-Lafuenteet al., 1998). No entanto, o mesmo comportamento é observado quando há o aumento do grau de hidrofilicidade dos suportes para valores mais expressivos. Nesse caso, é possível que a difusão do substrato para o interior do suporte seja facilitada, havendo um consequente aumento de atividade 
enzimática. Ao analisar a atividade de esterificação, Figura 3-B), o aumento do grau de hidrofobicidade provoca uma aumento da atividade do biocatalisador. Nos biocatalisadores mais hidrofóbicos, o centro ativo da CAL-B está, provavelmente, mais exposto, o que justifica as maiores atividades encontradas. Além disso, nas reações de esterificação, ao contrário das reações de hidrólise, a água não atua como um reagente e sim como um inibidor competitivo. Assim, os biocatalisadores mais hidrofóbicos possivelmente mantém a água mais afastada do sítio ativo, havendo um aumento da atividade de esterficação (condições aquo-restritas) (Freire, 1996).

Tabela 2 - Atividadesde hidrólise e de esterificação dos biocatalisadores produzidos.

\begin{tabular}{|c|c|c|}
\hline Suporte casca-núcleo & $\begin{array}{c}\text { Atividade de hidrólise do } \\
\left.\text { biocatalisador (UI/g } \mathrm{g}_{\text {bio }}\right)\end{array}$ & $\begin{array}{c}\text { Atividade de esterificação } \\
\text { do biocatalisador (UI/g }\end{array}$ \\
\hline PS-co-PDVB/PS & $2,5 \pm 0,2$ & $2030,5 \pm 153,7$ \\
\hline PS-co-PO/PS & $2,0 \pm 0,2$ & $240,0 \pm 153,7$ \\
\hline PS-co-PC/PS & $7,0 \pm 2,0$ & $237,8 \pm 153,7$ \\
\hline PS/PS & $3,2 \pm 1,6$ & $1258,0 \pm 153,7$ \\
\hline PS-co-PBV/PS & $4,1 \pm 2,7$ & $147,7 \pm 153,7$ \\
\hline
\end{tabular}

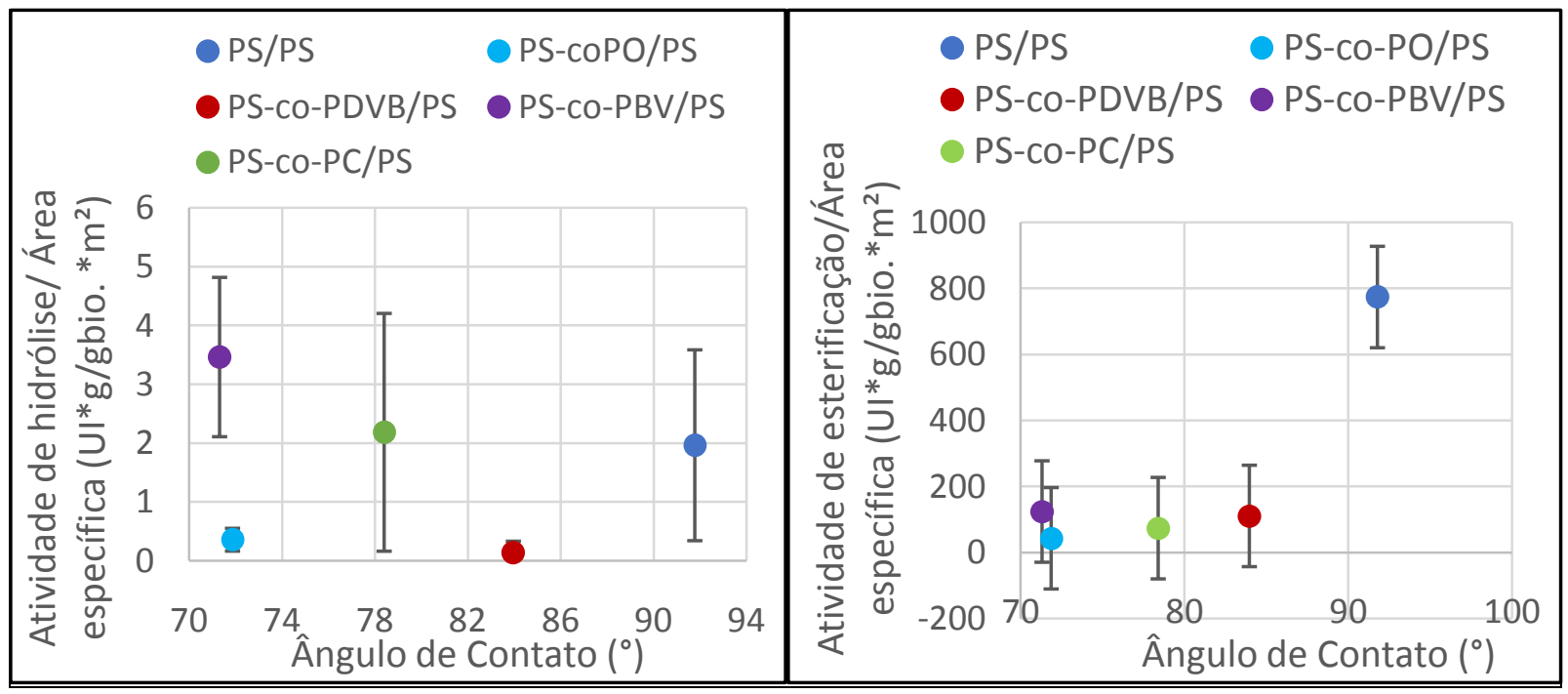

(A)

(B)

Figura 3-Influência do grau de hidrofobicidade dos suportescasca-núcleo na atividade dos biocatalisadores:A) atividade de hidrólise; B) atividade de esterificação.

\section{CONCLUSÃO}

Por tudo que foi apresentado, pôde-se observar que pequenas mudanças na composição da casca das partículas casca-núcleo provocam modificações expressivas na sua morfologia (área específica, diâmetro médio de poro) e grau de hidrofobicidade. Tais características morfológicas, por 


\section{9 a 22 de outubro de 2014 \\ Florianópolis/SC}

sua vez, afetam o comportamento cinético ao longo da imobilização.Foi possível notar também que o desempenho dos biocatalisadores produzidos depende não só da morfologia dos suportes empregados na imobilização da enzima, mas também da composição do meio reacional onde é empregado. Ao considerar as reações de hidrólise, aparentemente há uma região de mínima atividade do biocatalisador. No entanto, ao analisar as atividades de esterificação, o aumento do grau de hidrofobicidade dos suportes é acompanhado pelo aumento da atividade do biocatalisador correspondente. Os comportamentos observados resultam de dois efeitos antagônicos que afetam o desempenho do biocatalisador: maior exposição do sítio ativo das enzimas (suportes hidrofóbicos) ou menor (suportes hidrofílicos); presença mais significativa de limitações difusionais (reações de hidrólise) ou menos expressiva (reações de esterificação).

\section{REFERENCIAS}

BESTETI, M. D. Produção e caracterização de partículas casca-núcleo obtidas pela polimerização simultânea em suspensão e emulsão. Dissertação de mestrado, Universidade Federal do Rio de Janeiro, PEQ-COPPE, Rio de Janeiro, 2009.

CASTRO, H. F., ZANIN, G. M., MORAES, F. F., SÁ-PEREIRA, P.Imobilização de Enzimas e sua Estabilização. Em: Bon, E. P. S., Ferrara, M. A., Corvo, M. L.(eds).Enzimas em Biotecnologia: produção, aplicação e mercado, $1^{a}$ ed., capítulo 06, Rio de Janeiro: Editora Interciência, 2008.

CUNHA, A. G. Resolução cinética de derivados de mio-inositol catalisada por lipases. Tese de doutorado, Universidade Federal do Rio de Janeiro, Instituto de Química, Rio de Janeiro, 2011.

CUNHA, A. G.; BESTETI, M. D.; MANOEL, E. A.; DA SILVA, A. T.; ALMEIDA, R. V.; SIMAS, A. B. C.; FERNANDEZ-LAFUENTE, R.; PINTO, J. C.; FREIRE, D. M. G. Preparation of core-shell polymer support to imobilize lipase B from Candida antarctica. J. Mol. Catal. B: Enzym., v. 100, p. 59-67, 2014.

FERNANDEZ-LAFUENTE, R.; ARMISÉN, P.; SABUQUILLO, P. Imobilization of lipases by selective adsorption on hydrophobic supports. Chem. Phys. Lipids., v. 93, p. 185-197, 1998.

FREIRE, D. M. Seleção de microorganismos lipolíticos e estudo da produção de lipase por Penicillium restrictum. Tese de doutorado, Universidade Federal do Rio de Janeiro, 1996.

LENZI, M. K., SILVA, F. M., LIMA, E. L., PINTO, J. C. Semibatch Styrene Suspension Polymerization Processes. J. Appl. Polym. Sci., v. 89, p. 3021-3038, 2003.

PINTO, M. C. C. Influência de Variáveis Operacionais na Morfologia de Suportes Enzimáticos Preparados por Polimerização em Suspensão-Emulsão. Dissertação de mestrado, Universidade Federal do Rio de Janeiro, PEQ-COPPE, Rio de Janeiro, 2013.

UPPENBERG, J.; HANSEN, M.T.; PATKAR, S.; JONES, T. A. The sequence, crystal structure determination and refinement of two crystal forms of lipase B from Candida antarctica. Structure 2, p. 293-308, 1994.

VILlENEUVE, P., MUDERHWA, J. M., GRAILlE, J. Customizing lipases for biocatalysis A survey of chemical, physical and molecular biological approaches. J. Mol. Catal. B: Enzym., v. 9, p. 113-148, 2000. 


\section{ANEXO}

Todas as referências solicitadas pelos revisores foram adicionadas ao corpo do texto e na seção Referências, incluindo as referênciassolicitadas, correspondentes à metodologia experimental de dosagem da atividade de hidrólise e de esterificação dos biocatalisadores.

As referências utilizadas para determinação da metodologia experimental de dosagem da atividade de hidrólise e de esterificação dos biocatalisadores foram:

CUNHA, A. G. Resolução cinética de derivados de mio-inositol catalisada por lipases. Tese de doutorado, Universidade Federal do Rio de Janeiro, Instituto de Química, Rio de Janeiro, 2011.

CUNHA, A. G.; BESTETI, M. D.; MANOEL, E. A.; DA SILVA, A. T.; ALMEIDA, R. V.; SIMAS, A. B. C.; FERNANDEZ-LAFUENTE, R.; PINTO, J. C.; FREIRE, D. M. G. Preparation of core-shell polymer support to imobilize lipase B from Candida antarctica. J. Mol. Catal. B: Enzym., v. 100, p. 59-67, 2014.

PINTO, M. C. C. Influência de Variáveis Operacionais na Morfologia de Suportes Enzimáticos Preparados por Polimerização em Suspensão-Emulsão. Dissertação de mestrado, Universidade Federal do Rio de Janeiro, PEQ-COPPE, Rio de Janeiro, 2013. 